\title{
Quantifying Political Self-Organization in Social Media. Fractal patterns in the Spanish 15M movement on Twitter
}

\author{
Miguel Aguilera ${ }^{1,3 *}$, Ignacio Morer ${ }^{1,3}$, Xabier E. Barandiaran ${ }^{2,3}$ and Manuel G. Bedia ${ }^{1}$ \\ ${ }^{1}$ ISAAC, Dept. of Informatics, Universidad de Zaragoza, Spain. \\ ${ }^{2}$ IAS-Research Centre for Life, Mind, and Society \& Dept. of Philosophy \& \\ University School of Social Work, UPV/EHU University of the Basque Country, Spain. \\ ${ }^{3}$ DatAnalysis15M Research Network \\ * miguel.academic@maguilera.net
}

\begin{abstract}
The objective of this work is to better analyse and understand social self-organization in the context of social media and political activism. More specifically, we centre our analysis in the presence of fractal scaling in the form of $1 / f$ noise in different Twitter communication networks related to the Spanish $15 \mathrm{M}$ movement. We show how quantitative indexes of brown, white and pink noise correlate with qualitatively different forms of social coordination of protests: rigidly organized protests (brown noise), reactive-spontaneous protests (white noise) and complex genuinely self-organized protests (pink noise). In addition, pink noise processes present correlations that reach much further in time, maintaining a dynamical coherence that last several days, and also show a balance between mean distance and clustering coefficient within the interaction network.
\end{abstract}

\section{Introduction}

Artificial Life models have helped identify processes of self-organization in the living domain, opening up our scientific imagination and improving our theoretical insight into complex phenomena, ranging from chemical autocatalysis (Kauffman, 1993) to emergent collective intelligence (Bonabeau et al., 1999: Holland and Melhuish, 1999). Human social life has also been approached through Artificial Life techniques and theoretical lenses (Hemelrijk and Kunz, 2003) but it is now, with the rise of social media and digital data-mining, that a new door is opened for a genuine analysis and synthesis of human social life (Lazer et al. 2009).

One of the central topics of Artificial Life modelling has been the emergence of spontaneous structures or selforganized processes in nature: how can a distributed process organize into a collective pattern that maintains some organizational invariance? how does complexity arise spontaneously without an organizing centre? under which structural and environmental or boundary conditions is that possible? One can pose these very same questions in the realm of social life. In fact our social life is often subsumed under emergent collective patterns. A particularly interesting domain to put these type of questions to practical use is the realm of political organization and grassroots activism in social media. When is a process genuinely self-organized and participatory and when is it just an amplification of an organizing centre of power? Can "genuine" self-organization and the generation of a social "consciousness" be quantified?

The last years have witnessed an explosion of political activism based-on or catalysed-by social media. The Icelandic 'Kitchenware Revolution', Wikileaks 'Cablegate' and Anonymous' network defence, the 2011 Arab Spring, the Spanish 15M movement, the Occupy Movement... are but a few among the many examples of the increasing role played by social media in grassroots political organizing. While these and similar social movements differ in many important ways, there is one thing they share in common: they are all interwoven through autonomous communication networks supported by the Internet and wireless communication (Castells, 2012). Social media has provided the tools for creating horizontal and interactive communication networks, boosting enormously the possibilities for self-organized political processes.

The objective of this work is to better analyse and understand social self-organizing processes in the context of social media political activism. More specifically, we centre our analysis in the presence of fractal scaling in the form of $1 / f$ noise in different activist communication processes related to the Spanish 15M movement. We will start describing the context of the Spanish 15M movement and providing a short introduction to the theoretical tools used for our analysis. Next we describe the data, measures and results of the study, and we finally discuss the consequences and limitations of our work.

\section{M Movement and Social Media Channelled Self-Organization}

The presence of Internet and other digital media and the increasing use of multidirectional and interactive mass communication networks is starting to change radically the way societies organize themselves to constitute counter power or change power relationships with dominant institutions 
(Castells 2009). An important example of this phenomena is the protest movement that was born in Spain in May 15th 2011 (henceforth 15M movement). Initially, what would latter become the $15 \mathrm{M}$ movement was organized via social media preparing a massive demonstration without much visibility in traditional media. Unexpectedly, this social media work triggered a huge social movement involving between 6.5 and 8 million people (El País, 2011) that has abruptly changed the political life in Spain. Together with other parallel experiences (like the Arab Springs, or the Occupy movement), the $15 \mathrm{M}$ has renewed the communication strategies of previous social movements, now seen as obsolete due to new communicative practices adopted by a large segment of the population (Toret, 2012). Frequently, activists involved with the movement have described its organizational practices as dramatically different to their previous political experiences. These statements suggest that the movement cannot be described just as a group of well-defined formal organizations confronting established institutions. Instead, they often take borrowed dynamicist and complex systems metaphors and describe the organized movement as a swarm or an autopoietic network, with distributed and emergent properties (Sanchez Cedillo, 2012), a system governed by organismic cycles in which the activity of the system is coordinated through powerful synergies (Malo and Pérez, 2012) or as a 'self-organizing climate' which envelopes society (Fernández-Savater, 2012).

Unlike previous studies of network analysis of the $15 \mathrm{M}$ movement and the similar uprisings, the focus of this paper is on characterizing more global aspects of self-organization processes and exploring indicators of the kind of emergent communication patterns. More specifically, we will focus on the constitution of the system as a coherent whole which can maintain a dynamic identity for a period of time. Since this type of self-organization into a coherent dynamic unit is hypothesized to be the core of mental life and neural organization (Van Orden et al. 2003), we want to explore the possible analogy with social life and political consciousness.

\section{Fractal Scaling and Self-Organization in complex networks}

One of the greatest challenges for the understanding of cognitive and social system is finding formalisms to understand how complex activity emerges from processes of multi-scale organization. During the last decade, different authors have proposed methods of fractal analysis as a solid candidate for this task (Dixon et al. 2012).

Fractal scaling is characteristic of critically self-organized systems (Bak et al. 1987). In these systems we can find an interesting mix between stability and instability creating complex structures of the variability of the system's activity. Thus, processes with fractal scaling present a constant relation between the size of their fluctuations and the scale in which they occur: systematically larger fluctuations for longer scales and smaller fluctuations for shorter scales. We often describe fractality in a process through its spectral density function $S(f)$, which in the case of fractal scaling exhibits the form:

$$
S(f) \propto f^{-\beta}
$$

where $f$ stands for the different frequencies in which the activity of the process takes place, and $\beta$ defines a log-linear relationship between the spectral power content at different scales of $f$. The presence of log-linear relationships in the spectral density suggests that the activity of the system is self-organized into a nested temporal structure, in which the different rates of activity of the components of the system are coupled into a coherent macroscopic whole. More specifically, $\beta$ shows what is the relative influence of each scale in the system. Different values of $\beta$ describe different relationships between the weight of fast, medium, and slow timescales in the composition of a self-organized system.

The analysis of the fractal coefficients of a system's activity has been widely used in neuropsychology for characterizing different states of interactivity among the component of a cognitive system, as well as to predict the emergence of new cognitive structures (Dixon et al., 2012).

More concretely, different values of the $\beta$ parameter allows us to characterize different types of processes:

- White noise $(\beta=0)$ describes fully random fluctuations with no correlations in time (processes with no memory). White noise processes show a strong dependence on short time scale events (scales with higher frequencies). White noise processes display fast changes in their activity but are unable to maintain structured and coherent patterns.

- Brown noise $(\beta=2)$ resembles a diffussion process with no correlation between increments, but with a strong dependencies between the position of one sample and the next, presenting a "memory" of previous events. It shows a strong dependence on long time evens, where small frequencies give a much greater contribution to the noise structure than the rest. Brown noise processes are able to maintain stable structural patterns, but they are unable to flexibly modify their activity when fast changes are required.

- Pink noise $(\beta=1)$ describes processes in which an equilibrium is found between the influence of short, medium, and long timescales. It finds an equilibrium between disordered states with high informational content (white noise) and states with strong memory but low informational content (brown noise). Pink noise processes display dynamics which can maintain stable patterns of activity while being able to flexibly regulate their level of activity.

Fractal behavior has been frequently found in biology, psychology and neuroscience during the last decade, and 
fractal analysis have been used for characterizing the underlying structure of system producing complex behaviour. In psychology, fractal coefficients have been successfully used for the better understanding of different types of atypical developmental conditions and the prediction of cognitive outcomes (Dixon et al. 2012). Also, deviations from $\beta=1$ fractal scaling, either toward white or brown noise, have been found in different health conditions as epilepsy ( $\mathrm{Ra}-$ mon et al., 2008), heart failure (Goldberger, 2002), developmental dyslexia (Wijnants et al. 2012b), among many other examples (Wijnants et al. 2012a).

Although there is still controversy about the meaning of fractal scaling and its origin (Van Orden et al., 2005; Diniz et al. 2011), one position in the debate is that fractal scaling is a characteristic manifestation of self-organizing systems, which reflects the balance between independent and interdependent activity of their components. In this sense, deviations from perfect $\beta=1$ pink noise would imply unbalancing this equilibrium in favour of either independent or interdependent activity, affecting the ability of a system to behave in a self-organized manner. Recent years have witnessed increasing empirical support for the idea that pink noise may result from the interaction of many ongoing processes over a multiplicity of interdependent scales (Kello et al., 2007; Dotov et al. 2010, Wijnants et al. 2009).

According to these ideas, the measured $\beta$ coefficient can account for the type of underlying structures of complex phenomena. The absence of long-range correlations when $\beta=0$ implies that the different processes composing the system are highly independent, provoking uncorrelated randomness in the systems activity. This describes a situation where the ongoing activity is not self-organized at all and there is not a coherent collective pattern emerging from their activity. On the other hand we have $\beta=2$ processes where slow timescales dominate over others. These systems will display highly predictable patterns which will strongly constrain the individual dynamics of the system components, strangling the self-organization in favour of rigid and inflexible collective dynamics. Finally, when $\beta=1$, slow and fast timescales are compensated, and the influence of independent and interdependent activity is perfectly balanced; the activity of the system is going to depend on the ongoing reciprocal interactions between timescales, finding an equilibrium between stability and spontaneity. As a result $\beta=1$ becomes an indicator of distributed self-organization in a coherent whole: different parts of the system (with their characteristic frequencies) appear globally coordinated in a reciprocally influencing manner.

\section{Methods}

Now that we have proposed an indicator for characterizing different self-organization processes, we wish to apply it to answer questions about different instances of grassroots political mobilization. What is the relationship between indi- vidual political activity and the collective global process? Are processes of political organization of the digital age spontaneous angry mobs or just mindless followers of popular topics? What is the degree of collective 'political consciousness' in social media-based mobilizations?

We now proceed to present different sets of data about a series of political events that we have classified according to qualitative experience from observation and a set some measures aiming to provide quantitative indicators for answering some of the questions above.

\section{Data and qualitative analysis}

We have collected Twitter data from different protests taking place during May 2012, one year after the start of the $15 \mathrm{M}$ movement. That month was chosen because of the high density of mobilizations, allowing us to compare different organizational processes taking place in the same short period (thus neutralizing the influence of contextual factors and differences due to large scale variations on composition and methods of the $15 \mathrm{M}$ movement through its historical evolution). We downloaded around 385,000 Twitter messages using 20 different hashtags (labels used in twitter to identify conversational topics). Following the advise of activists involved within the protests, hashtags were chosen as representative of different types of processes for mobilization and protest:

- Events related to an education strike taking place on May 22 (\#22M, \#HuelgaDeClase), which follows a more traditional pattern of organization, with unions and other centralized organizations leading protesters, a fixed schedule of pickets, events, demonstrations, and a more predictable process of escalation of the mobilization as the strike date approaches.

- A series of events coinciding with the anniversary of the start of the $15 \mathrm{M}$ movement (with the global label \#12M15M), from May 12 to May 15, consisting mainly on a series of previously planned demonstrations named through different labels (\#ALaPlaza12M, \#Es15M, \#YoVoy12M, \#Feliz15M). Some of these preplanned events evolved into a series of spontaneous and more creative actions, as a campaign against the Bank ' $\mathrm{La}$ Caixa' (\#LaCaixaEsMordor) that spontaneously turned into a camp in front of the Headquarters of La Caixa with daily casserole protests under the label (\#OccupyMordor).

- Some events correspond to planned actions or proposals launched by some participants of the movement and amplified by the rest of the network in a distributed and decentralized way. This has been a characteristic mode of functioning of the $15 \mathrm{M}$ movement, which avoids formal leadership and centralized organization in favour of a more diffuse organization exploiting the possibilities of social media. Some examples of this 
are \#PlandeRescateCiudadano, \#BankiaEsNuestra, \#15MSectorRadical, \#NurembergFinanciero or \#CierraBankia.

- One special case of the category above is the case of \#15MPaRato. A team of activists and lawyers launched a campaign aiming to file a lawsuit demanding that the former director of Bankia (an Spanish bank accused of being a major responsible for the Spanish financial crisis), Rodrigo Rato and the rest of the director's board, be held accountable for mismanagement and possible criminal behaviour. The campaign was cooperatively designed to synchronously seed the message through a group of well positioned nodes within the movement's network, aiming to work as "catalysts" to create a supporting community for the organization of a citizen's lawsuit. This strategy turned out to be very successful, the hashtag \#15MPaRato quickly became trending topic in Spain and it reached the first position between the global trending topics soon after, it also managed to collect $€ 15000$ of funding in less than $24 \mathrm{~h}$ and contacting with tens of Bankia's stockholders and employees willing to take part as witnesses for the trial.

- A last set of events correspond to fast and spontaneous reactions to unexpected incidents, such as the moment where the Spanish risk premium reached the symbolic value of 500 points (\#Prima500), the police eviction of a social centre that was central to the movement (\#LaRimaia), the eviction of the protesters camped in Sol square in Madrid during the \#12M15M protests (\#DesalojoSol), or the spontaneous demonstration that followed as reaction to this last eviction targeting Madrid's Stock Exchange Building (\#ALaBolsa).

The task is now to test whether fractal scaling analysis can provide a good quantitative index for these qualitatively different degrees of self organization of social communication and coordination expressed through Twitter.

\section{Detrended Fluctuation Analysis}

The detrended fluctuation analysis (DFA; Peng et al., 2000) is a method for determining the statistical self-affinity of a signal. In a nutshell, the DFA algorithm integrates the analysed time series and then divides it into boxes of equal length $n$. For each box and each value of $n$, a least squares line (the trend of the signal within that box) is fit to the data. For each box size $n$, the characteristic size of the fluctuation $F(n)$ is computed as the rms deviation between the integrated signal and its trend in each box. This computation is repeated for every value of $n$. Typically, $F(n)$ increases with $n$. A linear relationship on a log-log plot with slope $\alpha$ indicates the presence of fractal scaling in the analysed signal (Figure 1). Where $\alpha$ is an approximation of the Hurst exponent, and is related to the scaling in the Power Spectrum of the Fourier analysis being $\beta=2 \cdot \alpha-1$.

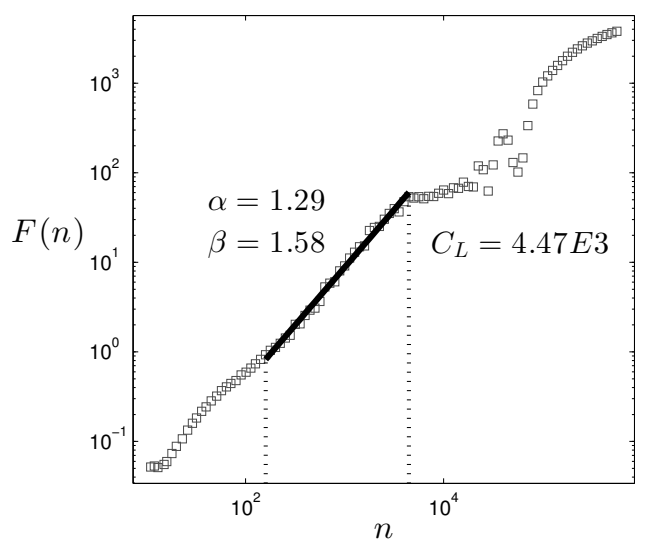

Figure 1: Detrended fluctuation analysis of the time series of tweets with the hashtag \#22M. The squares represent the values of $F(n)$ for each value of $n . C_{L}$ is the larger value of $n$ where the linear correlation still holds. The solid line represents the linear approximation of the 30 first samples under $C_{L}$. $\alpha$ represents the slope of the linear fit and $\beta$ its corresponding value of fractal scaling in the frequency spectrum. Copyright 2013 Miguel Aguilera Creative Commons Attribution-ShareAlike 3.0 Unported License

their common fractal dimension $F D$, which for spectral analysis takes the form $F D=0.1 \beta^{2}+0.4 \beta+1.5$, and for DFA $F D=0.4 \alpha^{2}-1.2 \alpha+2$.

For each hashtag, we created a time series composed of the number of messages that where written using that label at each instant of time (with a sampling period of $1 s$ ). The DFA algorithm was applied to the resulting series, obtaining the $F(n)$ values for each box size $n$. After that, we identified the larger value of $n$ where the shape of $F(n)$ stared to have a log-linear relationship. This value, which we will define as the correlation length of the process $\left(C_{L}\right)$ (Figure 11 , represents what is the larger scale where the fractal scaling holds. Over larger scales, the fractal relationships break down. We interpret this value as an indicator of how long a process can exist as a self-organized entity. Even if the process exists (i.e. the hashtag is used) for a longer time period, the emergence of coherent patterns of behaviour will not be larger that the value of $C_{L}$. Once identified, this temporal boundary of the self-organized process, we proceed to compute the fractal relations for smaller values of $n$. In order to avoid artefacts for small values of $n$, potentially due to the chosen sample rate (see Wijnants et al. 2013), we computed the value of $\alpha$ by fitting a least squares line of the 30 samples from $C_{L}-29$ to $C_{L}$ (Figure 1). The obtained trend was transformed into its corresponding value of $\beta$.

\section{Network Analysis}

After analysing the scale invariance of the different processes, we have completed our study by analysing the un- 
derlying networks generating those dynamics. We have taken the set of interactions between Twitter users (mentions, replies, retweets) using each of the hashtags to create a directed graph where each user is a node and interactions are represented by weighted edges. This graph represents the structure of interactions behind the communicational processes of each hashtag. For each network we have measured the following parameters and properties:

Degree of a node The degree of a node $k_{i}$ represents the number of incoming and outgoing connections of that node.

Clustering Coefficient It measures the connection density among the direct neighbours of a node. If we define $M_{i}$ as the number of connections between the neighbours of a node $i$ we can compute the mean clustering coefficient of a network with $N$ nodes as:

$$
C=\frac{1}{N} \sum_{i=1}^{N} \frac{M_{i}}{k_{i}\left(k_{i}-1\right)}
$$

A high Clustering coefficient implies a robust structure of the network.

Mean distance Distance $d(i, j)$ in a network measures the shortest path between two nodes $i$ and $j$. The mean distance of a network with $N$ nodes is computed as:

$$
L=\frac{1}{N(N-1)} \sum_{i, j} d(i, j)
$$

Small World Networks Small-world networks are defined as networks with high clustering coefficient and low mean distance (Watts and Strogatz, 1998). These networks have interesting properties which allow to easily find the shortest path between two nodes. Communication processes over small world structures will be much more robust and efficient. We can know if a network has small world properties from its clustering coefficient and its mean distance. Since the clustering coefficient and mean distance are strongly influenced by the size of the network, we have normalized both $C$ and $L$ by dividing them by the clustering coefficient and mean distance of a Erdös-Rényi random network with the same size and edge density $C_{r}$ and $L_{r}$. A network will have small world properties when $C_{L} \gg 1$ and $L_{r} \sim 1$.

Scale-Free Networks Small-world networks in nature often are found in the form of scale-free networks. Scale-free networks (Barabási and Bonabeau 2003) can naturally grow by preferential attachment of its nodes, resulting in networks in which some nodes called 'hubs' have a very high connectivity while the majority of the other nodes are poorly connected. Scale-free networks are characterized by a power law in its distribution of node degrees.

$$
P(k) \sim c \cdot k^{-\gamma}
$$

where $k$ and $\gamma$ are constants. All the networks analysed in this work displayed power law distributions of they degree distribution. Thus, we have computed the values of $\gamma$ for both the incoming and outgoing degree of connections using a linear regression on the logarithmic distribution of $P(k)$, obtaining two coefficients $\gamma_{\text {in }}$ and $\gamma_{\text {out }}$ for each network. The $\gamma_{i n}$ coefficient will represent the inequality in how the nodes act as sources of information. A high value of $\gamma_{i n}$ will imply that a few nodes are generating most of the information travelling through the network. In turn, $\gamma_{\text {out }}$ will represent the inequality in how the nodes propagate this information. A high value of $\gamma_{\text {out }}$ will mean that only a few nodes act as amplifiers of information in the network.

\section{Results}

Following the methods above, we have analysed the fractal exponent and the network properties for the 20 hashtags described above.

\section{Fractal Scaling}

In figure 2 we present the obtained values of $\beta$ and $C_{L}$. As we can see, the values of $\beta$ range between pink and brown noise (between 0 and 2 ), with some values very close to pink noise $(\beta=1)$. The results of $C_{L}$ also present quite different results, displaying more than two orders of magnitude between the shortest and larger temporal scope of the selforganized coordination.

In a closer inspection of the results, we can observe how different types of mobilization processes can be identified with different values of $\beta$. For example, the more rigidly organized process of the education strike (\#22M, \#HuelgaDeClase) displays values of $\beta$ closer to the smoother dynamics of brown noise. On the other hand, most of the "spontaneous" processes (\#ALaBolsa, \#LaRimaia, \#DesalojoSol), together with some of the messages amplified by the network (\#15MSectorRadical, \#NurembergFinanciero, \#LaCaixaEsMordor, \#BankiaEsNuestra). Finally, some processes seem to achieve an equilibrium between independent and interdependent dynamics and are quite close to pink noise (\#15MpaRato, \#12M15M and some of its related hashtags, \#PlandeRescateCiudadano, \#16M, \#Prima500, \#OccupyMordor), suggesting that these process reach some middle point between the spontaneousness of white noise and the stability of brown noise.

Figure 2 also shows a correlation between the values of $\beta$ and $C_{L}$. We have fitted the obtained values of $\beta$ and $C_{L}$ with a rational polynomial function of order two for both the numerator and denominator. The quality of the fit was measured by a $R^{2}$ coefficient of 0.69 . We observe how values of $\beta$ closer to one display much higher values of $C_{L}$, suggesting that when the process of self-organization reaches a dynamic equilibrium between independent and interdependent dynamics it spans into much larger temporal timescales. 
That is, independently of the real duration of the communication process, pink noise processes present correlations that reach much further in time, maintaining a dynamical coherence that lasts up to days in the cases with highest values of $C_{L}$.

\section{Network Properties}

Once the different types of self-organization processes have been classified according to their fractal exponents, we proceed to compare these results with the properties of their underlying networks.

Small-World Properties We have approximated the least squares second order curve of the normalized clustering coefficients and mean distances in relation to the obtained fractal coefficients $\beta$ (Figure 3). The results show that different types dynamics are related to different types of underlying network structures:

- White noise (low $\beta$ ): these processes have low values of $C / C_{r}$ and $L / L_{r}$, which means that their underlying networks have lower clustering coefficients (less robust structure) and shorter mean distances (faster information transfer). These results coincide with the idea of white noise processes as being spontaneous and viral but poorly organized processes which disintegrate quickly. Low clustering values and short distances also suggest that independent activity of the nodes has a higher influence on the dynamics of the system, since the activity of individual nodes travels fast and there is not a robust structure of stable communities.

- Brown noise (high $\beta$ ): these processes in turn present higher values of $C / C_{r}$ and $L / L_{r}$, meaning that their underlying networks are robust and well structured but have long communication paths. This is also compatible with the idea of brown noise processes being more robust but less flexible. A high clustering coefficient and long mean distance also suggests a bigger influence of interdependent activity, since communities are strong and the transmission of information trough the network is slow.

- Pink noise $(\beta \sim 1)$ : these processes find an equilibrium in which they have quite high values of $C / C_{r}$ and not very long mean distances $L / L_{r}$. Again, pink noise processes manage to have the best from white and brown noise processes, being robust at the same time as they are fast in the propagation of information.

Scale-Free properties We have approximated the least squares second order curve of the scale-free $\gamma_{i n}$ and $\gamma_{\text {out }}$ coefficients in relation to $\beta$ (Figure 4). We observe how there is a dependence between $\gamma_{i n}$ and $\beta$, finding that white noise processes present a more egalitarian distribution between the nodes that generate the contents of the communication,
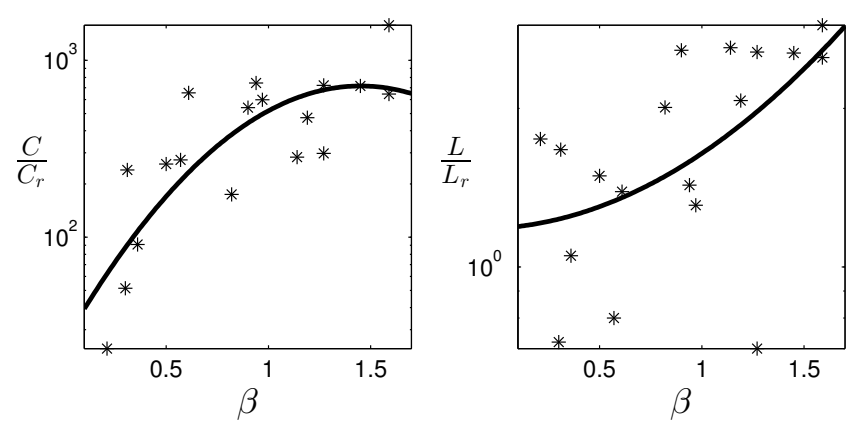

Figure 3: Values of the normalized clustering coefficient $C / C_{r}$ and mean distance $L / L_{r}$ with respect to the fractal coefficient $\beta$. The solid line represents a least squares second order fitting of the data. Copyright 2013 Ignacio Morer Creative Commons Attribution-ShareAlike 3.0 Unported License

while in brown noise processes there are fewer nodes leading the communication process. In the case of $\gamma_{\text {out }}$ it does not seem to have any strong correlation with $\beta$, suggesting that the role of the nodes as diffusers of the information is the same independently of the type of the self-organization process going on.
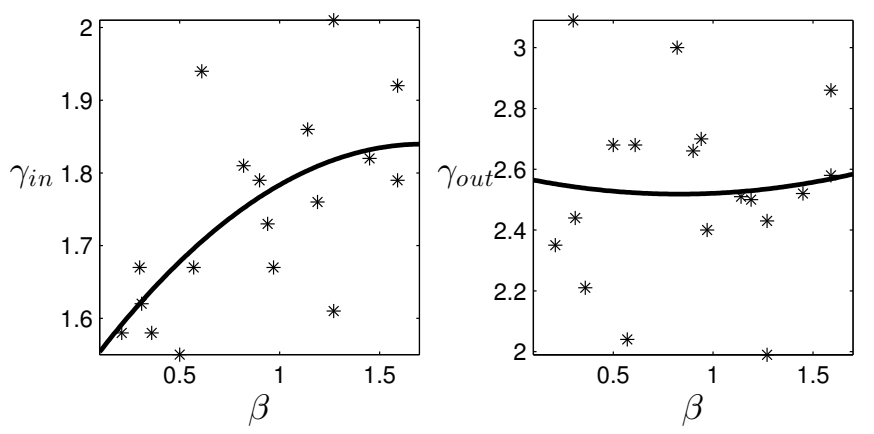

Figure 4: Values of the input and output scale-free coefficients $\gamma_{\text {in }}$ and $\gamma_{\text {out }}$ respect to the fractal coefficient $\beta$. The solid line represents a least squares second order fitting of the data. Copyright 2013 Ignacio Morer Creative Commons Attribution-ShareAlike 3.0 Unported License

\section{Discussion}

In previous sections we have presented fractal analysis as a candidate for quantifying and identifying different types of social coordination in communication networks. Concretely, we have proposed fractal scaling in in the frequency spectrum of the activity of a connected crowd as an indicator of how it constitutes itself as a collective social system trough ongoing interaction. We have measured fractal scaling as the relation between the amount of variability of the system 


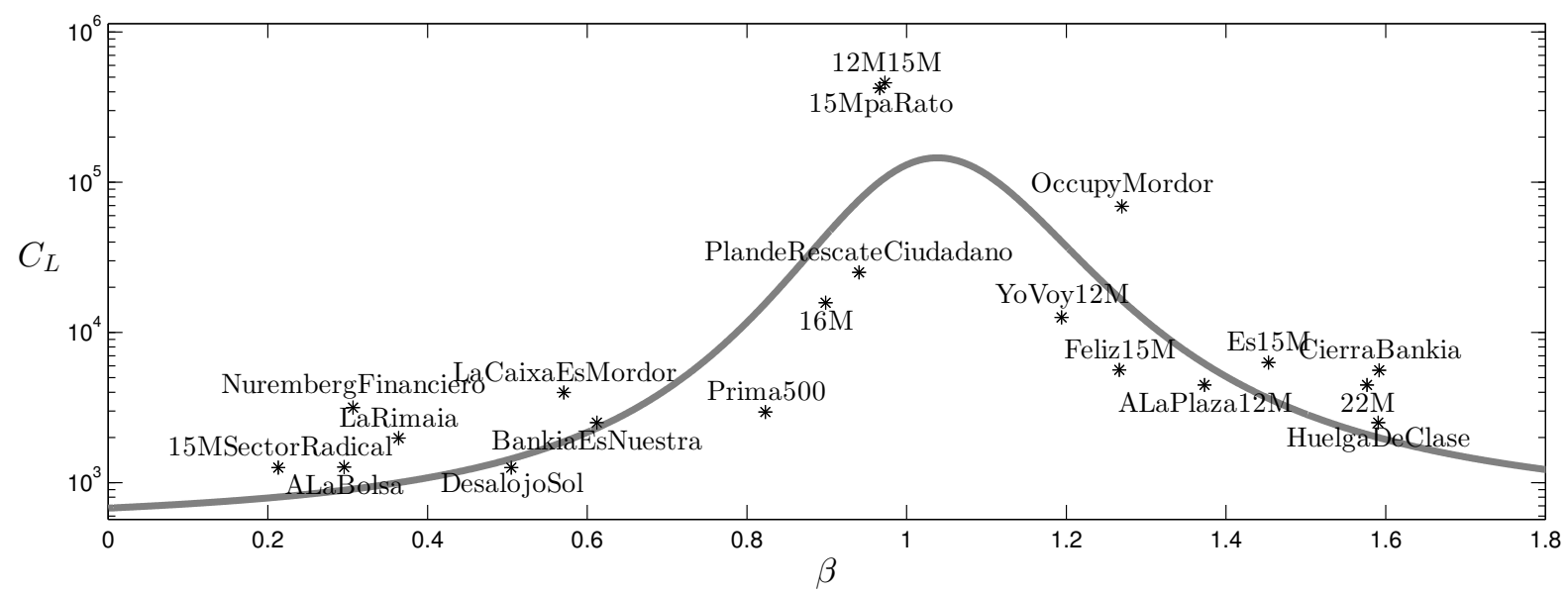

Figure 2: Values obtained for $\beta$ and $C_{L}$ (the latter measured in seconds) for the time series of messages with each hashtag. The solid line represents a rational polynomial fitting of the displayed values. Copyright 2013 Miguel Aguilera Creative Commons Attribution-ShareAlike 3.0 Unported License

at different temporal scales, obtaining a parameter $\beta$ that describes the fractal relations between the amount of activity in the system at different temporal scales. We have also measured other parameters like the temporal scope of the fractal scaling and properties of the network underlying the communicative activity.

Results have shown how processes which equilibrate the influence of the different temporal scales of activity (those with $\beta=1$ ) display properties that suggest that the ongoing process of self-organization is stronger than in other cases, as a larger fractal correlation length $C_{L}$ or more marked small world properties. In addition, the processes with $\beta \sim 1$ coincide with those social mobilizations having a mix of planned or stable development with more spontaneous or surprising turnarounds.

In contrast, processes with an unbalanced dynamical scaling, favouring either short-scale activity or long scale activity, do not display desirable properties for collective selforganization. The larger presence of fast or slow timescales implies that the system is giving preference to either independent or interdependent activity over the other. For example, we have seen how spontaneous reactions of social media activists to unexpected events tend to show white noise scaling, suggesting that the system is not really self-organized into a coherent unit of activity, but is rather the sum of the activities of a uncoordinated crowd reactively triggered by an external stimulus. On the other hand, we have seen how processes organized according to more rigid schemes, like as a strike, tend to brown noise scaling, suggesting that interdependent activity dominates the dynamics of the process, leaving no room for true self-organization as the individual dynamics are enslaved by the collective communicational process.
Despite the consistency of the present results, fractal scaling analysis must be taken carefully. Although fractal scaling is usually taken to emerge as a result of a self-organized processes, there is evidence suggesting that this is not always the case, and there are cases where fractal scaling can be the product of linear combinations processes without fractal scaling (e.g. with a linear superposition of random components acting on multiple time scales, see Hausdorff and Peng, 1996). To avoid this problem, some authors have suggested multifractal analysis to ensure the nonlinear nature of the ongoing interactions that build the self-organized process (Ihlen and Vereijken, 2010). In an extension of the present work (to be published) we have analysed the multifractal structure of some of the data presented here, confirming the relation between pink noise exponents and non-linear self-organized interactions we have claimed here.

Further progress in this direction might demand a more synthetic approach to test how artificial network topologies and communication dynamics yield different forms of noise and correlation length. Artificial Life techniques, like genetic algorithms, could be helpful to find optimal communication strategies in order to build genuine self-organized processes, including parametric analysis of the effect of variables such as degree of consensus, viral potential, external mass-media coverage, etc. Fractal scaling indexes could be used as fitness functions for these models.

\section{Conclusion}

The generalization of social media in everyday communication is a game changer for the analysis and understanding of self-organization in our social and political life. On the one hand, the use of digital communication networks has allowed to overcome some important limitations and diffi- 
culties for organizing large scale groups of people without a hierarchical structure (the series of political uprisings we have witnessed in the last 3 years is a good indicator of this potential). On the other hand, social media allows us to easily collect data from social interactions in different contexts. What is still missing is a deeper understanding of how social media network topology and dynamics correlates with different forms of collective action and self-organized coordination.

We have performed an analysis and comparison of 20 different communicative processes related to grassroots political mobilizations within the Spanish $15 \mathrm{M}$ movement in May 2012. A qualitative classification of these processes was shown to match the quantitative measurement of fractal scaling analysis of the message exchange time series. A balance between fast and slow temporal scales, described by a pink noise distribution, seems to boost the robustness and the life-span of genuine self-organized processes. Pink noise processes in social networks were also shown to be closely related with the stronger small-world properties of their underlying networks. Further analysis and modelling of social and political self-organization is required to support the hypothesis advanced in this paper but we might be witnessing the emergence of a pink noise revolution where it is the dynamics of social interaction (rather than the specific content being communicated) what matters. To say it paraphrasing Marshall McLuhan's celebrated motto, we might have entered an era where the noise is the message. And a science of Artificial (social) Life is perfectly suited to push this message forward.

\section{Acknowledgements}

M.A and M.G.B are supported in part by the project TIN201124660 funded by the Spanish "Ministerio de Ciencia e Innovación". M.A. currently holds a FPU predoctoral fellowship from the Spanish "Ministerio de Educación". X.E.B acknowledges funding from the research project "Autonomía y Niveles de Organización" financed by the Spanish Government (ref. FFI2011-25665) and IASResearch group funding IT590-13 from the Basque Government.

Specially, we would like to acknowledge our colleagues of DatAnalysis15M for their work and support.

This work is licensed under a Creative Commons AttributionShareAlike 3.0 Unported License

\section{References}

Bak, P., Tang, C., and Wiesenfeld, K. (1987). Self-organized criticality: An explanation of the 1/f noise. Phys. Rev. Lett., 59(4):381-384.

Barabási, A. and Bonabeau, E. (2003). Scale-free networks. Sci Am, 288(5):60-69.

Bonabeau, E., Dorigo, M., and Theraulaz, G. (1999). Swarm Intelligence: From Natural to Artificial Systems. Oxford University Press, U.S.A.

Castells, M. (2009). Communication power. Oxford University Press.

Castells, M. (2012). Networks of Outrage and Hope: Social Movements in the Internet Age. Wiley.

Diniz, A., Wijnants, M. L., Torre, K., Barreiros, J., Crato, N., Bosman, A. M. T., Hasselman, F., Cox, R. F. A., Van Orden, G. C., and Delignires, D. (2011). Contemporary theories of 1/f noise in motor control. Hum Mov Sci, 30(5):889905. PMID: 21196059.
Dixon, J. A., Holden, J. G., Mirman, D., and Stephen, D. G. (2012). Multifractal dynamics in the emergence of cognitive structure. Top Cogn Sci, 4(1):5162.

Dotov, D. G., Nie, L., and Chemero, A. (2010). A demonstration of the transition from ready-to-hand to unready-to-hand. PLOS ONE, 5(3):e9433.

El País, E. (2011). Hasta 8,5 millones de españoles apoyan el movimiento 15-m.

Fernández-Savater, A. (2012). Cómo se organiza un clima? http://blogs.publico.es/fueradelugar/1438/como-se-organiza-un-clima.

Goldberger, A. L. (2002). Fractal dynamics in physiology: Alterations with disease and aging. Proc Natl Acad Sci, 99(90001):2466-2472.

Hausdorff, J. and Peng, C.-K. (1996). Multiscaled randomness: A possible source of 1/f noise in biology. Phys Rev E, 54(2):2154-2157.

Hemelrijk, C. K. and Kunz, H. (2003). Introduction to special issue on collective effects of human behavior. Artif Life, 9(4):339341.

Holland, O. and Melhuish, C. (1999). Stigmergy, self-organization, and sorting in collective robotics. Artif Life, 5(2):173-202.

Ihlen, E. A. F. and Vereijken, B. (2010). Interaction-dominant dynamics in human cognition: Beyond 1/ fluctuation. J Exp Psychol Gen, 139(3):436-463.

Kauffman, S. A. (1993). The origins of order. Oxford University Press US.

Kello, C. T., Beltz, B. C., Holden, J. G., and Van Orden, G. C. (2007). The emergent coordination of cognitive function. J Exp Psychol Gen, 136(4):551-568. PMID: 17999570.

Lazer, D., Pentland, A., Adamic, L., Aral, S., Barabási, A.-L., Brewer, D., Christakis, N., Contractor, N., Fowler, J., Gutmann, M., Jebara, T., King, G., Macy, M., Roy, D., and Alstyne, M. V. (2009). Computational social science. Science, 323(5915):721-723. PMID: 19197046.

Malo, M. and Pérez, D. (2012). Latidos: el 15m y la revuelta. In Democracia Distribuida. Miradas de la Universidad Nomada al 15M.

Peng, C. K., Hausdorff, J. M., and Goldberger, A. L. (2000). Fractal mechanisms in neuronal control: human heartbeat and gait dynamics in health and disease. In Self-Organized Biological Dynamics and Nonlinear Control. Cambridge University Press.

Ramon, C., Holmes, M. D., Freeman, W. J., McElroy, R., and Rezvanian, E. (2008). Comparative analysis of temporal dynamics of EEG and phase synchronization of EEG to localize epileptic sites from high density scalp EEG interictal recordings. Conf Proc IEEE Eng Med Biol Soc, 2008:4548-4550. PMID: 19163727

Sanchez Cedillo, R. (2012). El 15M como insurrección del cuerpo-máquina. In Democracia Distribuida. Miradas de la Universidad Nomada al 15M.

Toret, J. (2012). Una mirada tecnopolítica sobre los primeros días del 15M. In Democracia Distribuida. Miradas de la Universidad Nómada al 15M.

Van Orden, G. C., Holden, J. G., and Turvey, M. T. (2003). Self-organization of cognitive performance. J Exp Psychol Gen, 132(3):331-350. PMID: 13678372.

Van Orden, G. C., Holden, J. G., and Turvey, M. T. (2005). Human cognition and 1/f scaling. J Exp Psychol Gen, 134(1):117-123. PMID: 15702967.

Watts, D. J. and Strogatz, S. H. (1998). Collective dynamics of small-world networks Nature, 393(6684):440-442.

Wijnants, M. L., Bosman, A. M. T., Hasselman, F., Cox, R. F. A., and Van Orden, G. C. (2009). 1/f scaling in movement time changes with practice in precision aiming. Nonlinear Dynamics Psychol Life Sci, 13(1):79-98. PMID: 19061546.

Wijnants, M. L., Cox, R. F. A., Hasselman, F., Bosman, A. M. T., and Orden, G. V. (2013). Does sample rate introduce an artifact in spectral analysis of continuous processes? Front. Physio, 3:495.

Wijnants, M. L., Cox, R. F. A., Hasselman, F., Bosman, A. M. T., and Van Orden, G. (2012a). A trade-off study revealing nested timescales of constraint. Front Physiol, 3:116. PMID: 22654760.

Wijnants, M. L., Hasselman, F., Cox, R. F. A., Bosman, A. M. T., and Van Orden, G (2012b). An interaction-dominant perspective on reading fluency and dyslexia. Ann Dyslexia, 62(2):100-119. PMID: 22460607. 